\title{
When one-two-three beats two-one-three: Tracking the acquisition of the verbal number sequence
}

\author{
Amandine Van Rinsveld ${ }^{1}$ (D) $\cdot$ Christine Schiltz $^{2} \cdot$ Steve Majerus $^{3} \cdot$ Michel Fayol $^{4}$
}

Published online: 3 January 2020

(C) The Psychonomic Society, Inc. 2020

\begin{abstract}
Learning how to count is a crucial step in cognitive development, which progressively allows for more elaborate numerical processing. The existing body of research consistently reports how children associate the verbal code with exact quantity. However, the early acquisition of this code, when the verbal numbers are encoded in long-term memory as a sequence of words, has rarely been examined. Using an incidental assessment method based on serial recall of number words presented in ordered versus non-ordered sequences (e.g., one-two-three vs. two-one-three), we tracked the progressive acquisition of the verbal number sequence in children aged 3-6 years. Results revealed evidence for verbal number sequence knowledge in the youngest children even before counting is fully mastered. Verbal numerical knowledge thus starts to be organized as a sequence in longterm memory already at the age of 3 years, and this numerical sequence knowledge is assessed in a sensitive manner by incidental rather than explicit measures of number knowledge.
\end{abstract}

Keywords Counting $\cdot$ Development $\cdot$ Verbal number sequence $\cdot$ Number words

\section{Introduction}

Although some numerical skills are deeply rooted in nonverbal human cognition, the exact quantity of large item sets can only be grasped using number words and symbols (Izard, Pica, Spelke, \& Dehaene, 2008; Pica, Lemer, Izard, \& Dehaene, 2004). The learning of those number labels starts with early counting activities, which thus represent an important precursor of formal mathematical learning (e.g., Martin,

\section{Highlights}

- Early knowledge of the verbal number sequence is difficult to assess.

- We developed an incidental measure for verbal number sequence knowledge.

- Serial recall tasks for ordered versus non-ordered number sequences were used.

- Better recall of ordered number sequences even before counting is fully mastered.

- Novel and sensitive method for assessing early number knowledge.

Amandine Van Rinsveld am.vanrinsveld@gmail.com

1 Center for Research in Cognition and Neuroscience, Université libre de Bruxelles, 50 av. F. Roosevelt, 1050 Bruxelles, Belgium

2 Institute of Cognitive Science and Assessment, Education, Culture, Cognition and Society Research Unit, University of Luxembourg, 11, Porte des Sciences, L-4366 Esch-sur-Alzette, Luxembourg
Cirino, Sharp, \& Barnes, 2014; Mundy \& Gilmore, 2009). When children start learning to count, number names are not yet related to quantities or to children's preverbal numerical abilities (Huntley-Fenner \& Cannon, 2000; Wynn, 1992). Indeed, children first acquire number names as a verbal routine, and these number names cannot be used independently from the learned sequence context and they are not yet associated with numerosity (Le Corre, Vandewalle, Brannon, \& Carey, 2006; Sarnecka \& Carey, 2008). Only later do children 
learn to associate the number names with the corresponding quantities (Gallistel \& Gelman 1992; Gelman \& Gallistel, 1978; Laski \& Siegler, 2007). A large set of studies has examined how children progressively link both the verbal and the symbolic codes to numerical quantity across development (e.g., Benoit, Lehalle, Molina, Tijus \& Jouen, 2013; De Smedt, Noël, Gilmore, \& Ansari, 2013; Mussolin, Nys, Leybaert, \& Content, 2015), but very few studies have investigated how children learn the verbal number names. The current paper focuses on this early stage of number acquisition. Existing evidence of this early verbal number knowledge comes from explicit counting tasks (i.e., producing the verbal number sequence upon request), which are often imprecise and unreliable. Because explicit counting tasks only partially reflect the level of knowledge of the verbal number sequence, we designed an incidental method based on immediate serial recall of number sequences for assessing verbal number sequence knowledge in a sensitive manner already at an early developmental age.

In the first place, numbers are learned as a sequence of words, but across development the representations associated with this sequence knowledge undergo qualitative changes. Fuson (1988) described five successive stages of learning of the verbal number sequence from the string level where the sequence is recalled as a unique structure that is not associated with numerical meaning or even divisible, to the bidirectional chain level where the number words can be produced forward and backward and used as the basis for arithmetic problem solving. Fuson (1988) proposed that children could simultaneously be at different stages of verbal number acquisition depending on the range of numbers (e.g., fifth stage for numbers from 1 to 10 but third stage for numbers from 20 to 100), going from an acquisition phase where they learn the words to an elaboration phase where they are able to manipulate the words. Recent work showed that these two phases may overlap across development (Gould, 2017), and that children iterate knowledge about the smaller numbers to larger numbers up to 100 . This overlap makes it difficult to isolate the different stages of verbal number learning.

Since the seminal work of Fuson, not much attention has been paid to the very first step of verbal number sequence knowledge although it constitutes a fundamental step of counting acquisition and by consequence a central aspect of numerical cognition. One of the reasons for this gap may be that numbers have been considered as a block of verbal knowledge that, once acquired, is simply transferred to the corresponding numerical quantities. However, Barrouillet et al. (2010) showed that children possess some implicit knowledge about two- or three-digit numbers in the counting list long before being able to recite them explicitly as when asked to count up to 1,000 . The hypothesis that children would also possess some similar implicit knowledge about the first verbal numbers in the counting list (1-19) has never been empirically tested and validated by a detailed examination of the progressive elaboration of the verbal number sequence.

Critically, before children acquire an understanding of the cardinal relations between number words (e.g., " 6 is one smaller than 7"), they can recite the number chain (Fuson, Richards, \& Briars, 1982; Wynn, 1992) and also possess some knowledge about the ordinal relations between number words (e.g., "6 comes before 7”; Colomé \& Noël, 2012; Lyons \& Beilock, 2009). This indicates that children may acquire representations of the verbal labels of numbers and their order in a specific range before being able to explicitly count in that range. However, it is difficult to specifically track how well a number sequence is encoded in long-term memory without putting any emphasis on the meaning of the number words, as it is for instance the case with explicit counting tasks or with questions on the numbers' relative position (e.g., " 6 comes before 7"). Because of this difficulty in isolating the verbal number sequence knowledge from other properties of counting, little is known about the implicit knowledge of the verbal sequence itself and its impact on explicit counting skills and later successful numerical development.

The current study aimed at providing a novel and implicit measure of verbal number sequence knowledge and advancing our understanding of the development of this knowledge in young children aged 3-6 years. We compared four groups of children ranging from 3 to 6 years in different conditions of immediate serial recall of number word lists. We compared the children's ability to recall ordered as opposed to non-ordered lists of verbal numbers (e.g., 7-5-6 vs. 5-6-7). The list length varied from three to five items to go beyond the children's working memory span, and the numbers to be recalled ranged from 1 to 19 to go beyond the children's current counting abilities. The difficulty in recalling non-ordered lists should increase with increasing list length as performance will rely exclusively on the children's working memory abilities (Gathercole, 1999). Critically, recall performance for ordered lists should be facilitated if children already possess corresponding knowledge about the order of verbal number labels in long-term memory. In other words, we propose using a working memory task here in order to incidentally assess the knowledge children have about the verbal number chain.

The rationale of this study is based on the fact that working memory interacts with long-term memory knowledge in an obligatory and automatic manner. Given the inherent difficulty of working memory tasks, with a capacity limit of up to four to five separate items in adults (Cowan, 2001), any long-term knowledge structure that is available will be used to overcome these limitations of working memory. For example, while working memory span for novel words rarely exceeds two to three non-words (equalling to four to five syllables), word span is about four to five items because the syllables defining a familiar word will be chuncked together as a single lexical 
word form by our linguistic knowledge base (Hulme, Maughan, \& Brown, 1991; see Majerus, 2019, for a review). Furthermore, this knowledge also involves the sequential aspects of linguistic processing: Syllable sequences that are composed of phonemes that frequently co-occur in a given language will lead to higher recall performance than syllable sequences composed of phonemes that co-occur less frequently (Gathercole, Frankish, Pickering, \& Peaker, 1999; Majerus, Van der Linder, Mulder, Meulemans, \& Peters, 2004; Majerus, Perez, \& Oberauer, 2012). The influence of longterm knowledge on verbal working memory is highly robust since it is observed at any developmental stage (Gathercole et al., 1999; Majerus \& Van der Linden, 2003). It is also independent of strategy use as long-term memory knowledge influences verbal working memory performance when memoranda are presented at a very fast encoding speed and the use of item-level or list-level elaborative processes are prevented (Kowialiewski \& Majerus, 2018). By capitalizing on this fundamental property of verbal working memory, the aim of this study was to probe the existence of implicit verbal number sequence knowledge and to determine the developmental stage at which this knowledge emerges. As soon as this knowledge is available, it should have an obligatory impact on verbal working memory performance and lead to higher recall performance for ordered than non-ordered number lists.

\section{Method}

\section{Participants}

We recruited a total sample of 82 children composed of $203-$ year-olds (11 females; mean age of 42 months; $\mathrm{SD}=3.2$ months), 214 -year-olds (12 females; mean age of 54 months; $\mathrm{SD}=2.4$ months), 215 -year-olds ( 10 females; mean age of 67 months; SD $=3.4$ months) and 20 6-year-olds (12 females; mean age of 75 months; SD $=2.5$ months). All participants were native French speakers from homogeneous middle socio-economic backgrounds recruited in kindergarten and primary schools. Informed consent was obtained for all participants and their parents.

\section{Stimuli}

The verbal number sequences to recall consisted of trials of different lengths: three, four, or five number words. We presented eight sequences of each length. Half of the sequences were composed of number words ranging from one to nine (small numbers) with the other half ranging from 11 to 19 (large numbers). Although the number words from 17 to 19 in French could be translated as ten-seven, ten-eight, and ten-nine, respectively, previous studies on French number words did not show any difference in processing between 11-16 and 17-19
(Jarlégan et al., 1996; Seron \& Fayol, 1994). We thus considered all numbers from 11 to 19 as large numbers. Each sequence was presented twice: Once as a canonically ordered sequence (e.g., 3 $-4-5$ ) and once as a non-ordered sequence (e.g., $5-3-4$ ), resulting in a total of 48 sequences (see complete list of stimuli in the Supplementary Material section). The same number word could not appear more than once within a sequence (e.g., no sequences like 4-9-9 or 4-7-4). The order of the items in the non-ordered sequences was pseudo-randomized by avoiding inverted ordered sequences (e.g., $7-6-5$ ) and discontinuous ordering (e.g., $2-3-8$ or $2-4-6$ ).

\section{Procedure}

Children were instructed to recall each list immediately after presentation exactly as they had heard it. All types of lists were administered to all children but the experimenter gave no hint or information about the characteristics of the lists. All stimuli had been pre-recorded and were presented on a computer using E-Prime 1.x software (Psychology Software Tools, Pittsburgh, PA, USA); all stimuli within a sequence were presented with an inter-stimulus interval of $1 \mathrm{~s}$. The experimenter started the presentation of each list by pressing a button. Before the start of the task, the participants had to recall a sequence of two letters to ensure their understanding of the task instruction. Next, three trials of two number word items were presented as further practice trials. After this practice phase, the experimental trials started and were presented with increasing length. The small- or large-number sequences and the ordered and non-ordered sequences of the same length were presented in semi-random succession with the following rule: two opposite trials of the same condition could not follow each other (e.g., $5-6-7$ and then $15-16-17$ were not allowed). We summed the number of sequences correctly recalled by each participant separately for the different types of trials (non-ordered vs. ordered, small vs. large numbers, and three vs. four vs. five items) resulting in a score ranging from 0 to 4 . This score was transformed in proportions.

After the recall task, counting ability was tested by asking the children to count as far as they could and by stopping the task when children reached 30 . No additional prompts were given when the child stopped counting. The score was the largest number to which the children could count before making any errors. The total experiment lasted 20-30 min.

\section{Results}

We ran an ANOVA on number list immediate serial recall performance with order (ordered vs. non-ordered sequences), length (three-item, four-item, or five-item sequences), and range (small 1-9 vs. large 11-19 numbers) as within-subject factors, and age group as between-subject factor. 
We observed a main effect of length, $F(2,156)=293.65$, $p$ $<.001, \eta^{2}=.79$, with shorter sequences being better recalled than longer sequences, a main effect of order, $F(1,78)=$ $149.79, p<.001, \eta^{2}=.67$, with ordered sequences being better recalled than non-ordered sequences, and an effect of range, $F(1,78)=73.82, p<.001, \eta^{2}=.48$, with small-range numbers being better recalled in general. We also observed a main effect of age group, $F(3,78)=30.24, p<.001, \eta^{2}=.53$, showing increasing recall performance as a function of age group.

Furthermore, there was a significant age-by-length interaction, $F(6,156)=22.02, p<.001, \eta^{2}=.46$, and a significant age-by-order interaction, $F(3,78)=13.63, p<.001, \eta^{2}=.66$. These interactions were characterized by an increase in the list length effect and the numerical order effect as a function of age group with age $\left(\eta^{2}=.40, .67, .85, .92\right.$ for the length effect as a function of age group; $\eta^{2}=.22, .61, .70, .79$ for the numerical order effect as a function of age group) ( see Fig. 1).

Moreover, there was also a significant interaction between range and order, $F(1,78)=21.41, p<.001, \eta^{2}=.22$; its decomposition through pairwise comparisons revealed that the difference in recall performance between small and large numbers was more important in ordered than in non-ordered sequences, $F(1,78)=72.96, p<.001, \eta^{2}=.48$, and $F(1,78)=$ $13.43, p<.001, \eta^{2}=.15$, for small and large number, respectively (see Fig. 2).

Further, we observed a significant interaction between order and length, $F(2,156)=4.90, p=.009, \eta^{2}=.59$, and a significant order $\times$ length $\times$ group interaction : $F(6,156)=$ $4.64, p<.001, \eta^{2}=.15$. Decomposition of these interactions revealed better recall for ordered sequences than non-ordered sequences for all three sequence lengths (three-item lists, $F(1,78)=33.52, p<.001, \eta^{2}=.30$; four-item lists, $F(1,78)$ $=63.42, p<.001, \eta^{2}=.45$; five-item lists, $F(1,78)=89.904, p$ $\left.<.001, \eta^{2}=.54\right)$, but also that the effect of order increased as a function of age group, but only for four-item and five-item sequences (three-item lists, $F(3,78)=1.31, p=.28, \eta^{2}=.05$; four-item lists, $F(3,78)=8.43, p<.001, \eta^{2}=.25, F(3,78)=$ 14.23, $p<.001, \eta^{2}=.35$ ) (see Fig. 3).

In order to directly compare number sequence knowledge as revealed by the immediate serial recall task to standard counting task performance, we examined immediate serial recall performance for ordered versus non-ordered sequences in children who could not reliably count within the range of the numbers used for the recall task (1-19). Counting ability increased with age, such that 3 -year-olds counted on average up to $7 \pm 5,4$-year-olds to $17 \pm 9,5$-year-olds to $28 \pm 3$, and 6year-olds to $29 \pm 0.5$. When selecting only the children who were not yet able to count up to 20 (a total of 31 children, among which were all 3-year-olds and eight 4-year-olds), we still observed better serial recall for ordered than non-ordered sequences, $t(30)=4.31, p<.001$. When limiting the analysis to the children who were not yet able to count up to 10 , serial recall was still significantly better for ordered sequences than for non-ordered sequences, and this was the case both for small $(1-10), t(18)=2.72, p=.014$, and for large numbers $(11-19), t(18)=2.52, p=.021$. Ordered sequences are thus better recalled than non-ordered sequences, even in children who show no reliable explicit knowledge of the verbal number sequence.

Finally, we assessed whether the serial recall performance for ordered sequences could predict counting skills using multiple linear regressions. Indeed, when asked to count as far as they could, 17 out of the 6-year-old children, 11 out of the 5 year-old children, and two out of the 4-year-old children could count up to 30; none of the 3-year-old children could count up to 30 (see above for average counting performances in each group). We predicted performance on the counting task by recall performance for ordered sequences, after controlling for general serial recall abilities by including recall performance for non-ordered sequences as a first predictor. We observed that although the non-ordered recall regressor already predicted counting skills, $\mathrm{R}^{2}=.44, F(1,79)=61.92, p<.001$, standardized regression coefficient $\beta=.66, p<.001$, the addition of the ordered recall regressor significantly improved the prediction of counting abilities, $\mathrm{R}^{2}=.55, F(1,78)=19.23$, $p<.001$, standardized regression coefficient of ordered sequences on counting $\beta=.75, p<.001$. Ordered recall performance thus predicted a unique amount of variance in counting skills, after controlling for general working memory abilities via the non-ordered recall regressor. To test whether the opposite was true, we ran the same model but introducing the ordered recall regressor at the first step and then adding the non-ordered recall regressor. While the ordered recall regressor significantly predicted counting skills, $\mathrm{R}^{2}=.55, F(1,79)=$ $96.65, p<.001$, standardized regression coefficient $\beta=.74, p$ $<.001$, this was not the case anymore for the non-ordered recall regressor when added at the second stage, $\mathrm{R}^{2}=.55$, $F(1,78)=47.72, p<.001$, standardized regression coefficient of non-ordered sequences on counting $\beta=-.01, p=.968$.

\section{Discussion}

In this study we used an immediate serial recall task for lists of number words in order to assess in an incidental and sensitive manner the development of verbal number sequence knowledge in children aged 3-6 years. This incidental assessment method for number knowledge allows assessment of children's verbal number sequence knowledge in an implicit manner that is complementary to traditional counting tasks. The method developed here may be particularly useful for assessing number sequence knowledge in very young children.

The incidental effect of number sequence knowledge was assessed by comparing recall for ordered versus non-ordered 

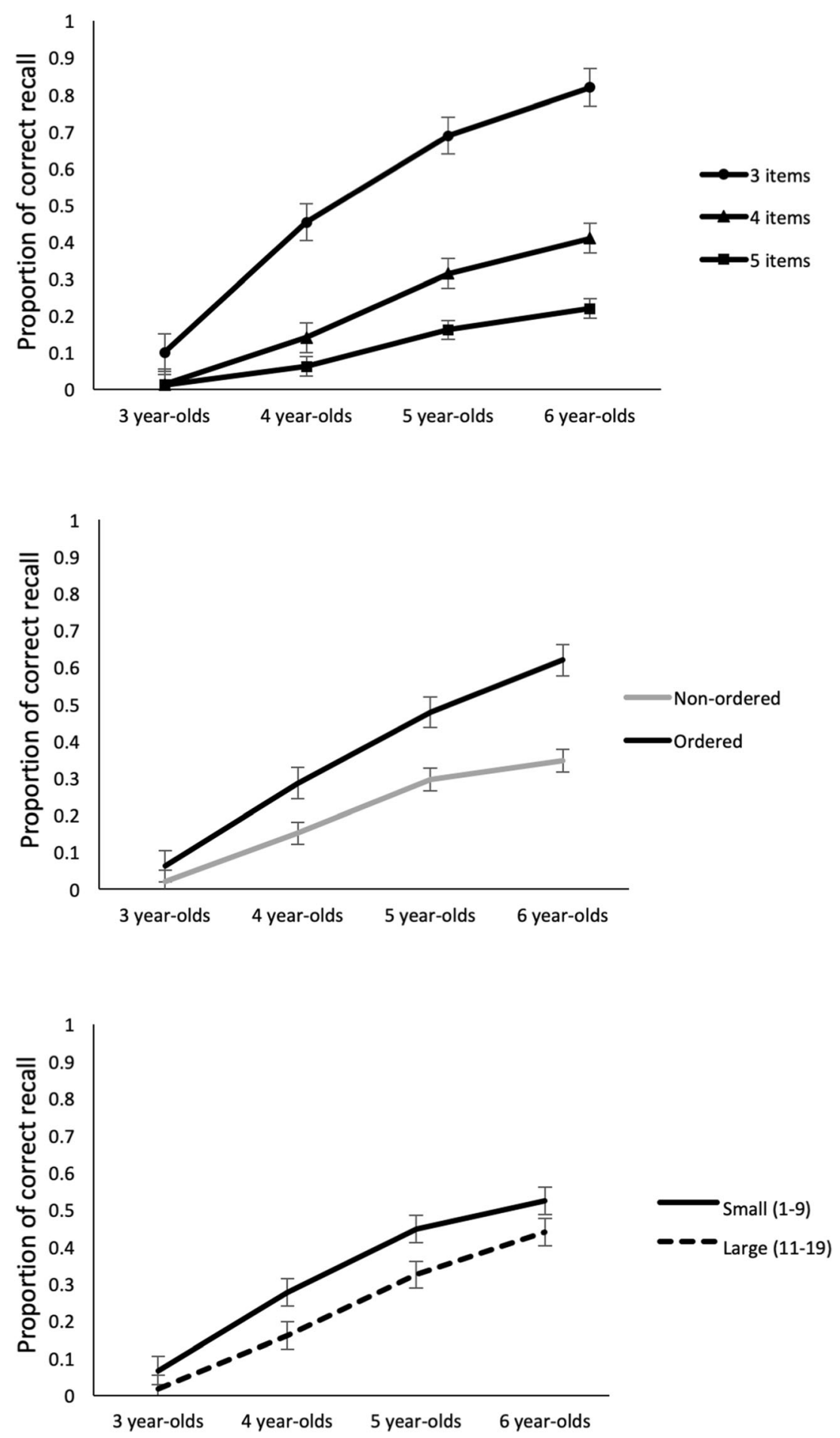

Fig. 1 Recall performance (accuracy) as a function of age group and list length (upper panel), numerical order (middle panel), or numerical range (lower panel). Error bars depict standard errors

sequences. We observed better immediate serial recall performance for ordered sequences in all age groups, and this effect also increased with age group. Furthermore, this effect was stronger for sequences involving small numbers as compared to large numbers in all age groups and suggests a greater familiarity for verbal sequence knowledge involving small numbers. This sustains Fuson's idea that different portions of the verbal number sequence can achieve distinct levels of establishment at a given moment (Fuson, 1988). Critically, the advantage of ordered over non-ordered recall was observed even in the youngest children who did not yet fully master counting as assessed by a traditional, explicit number- 


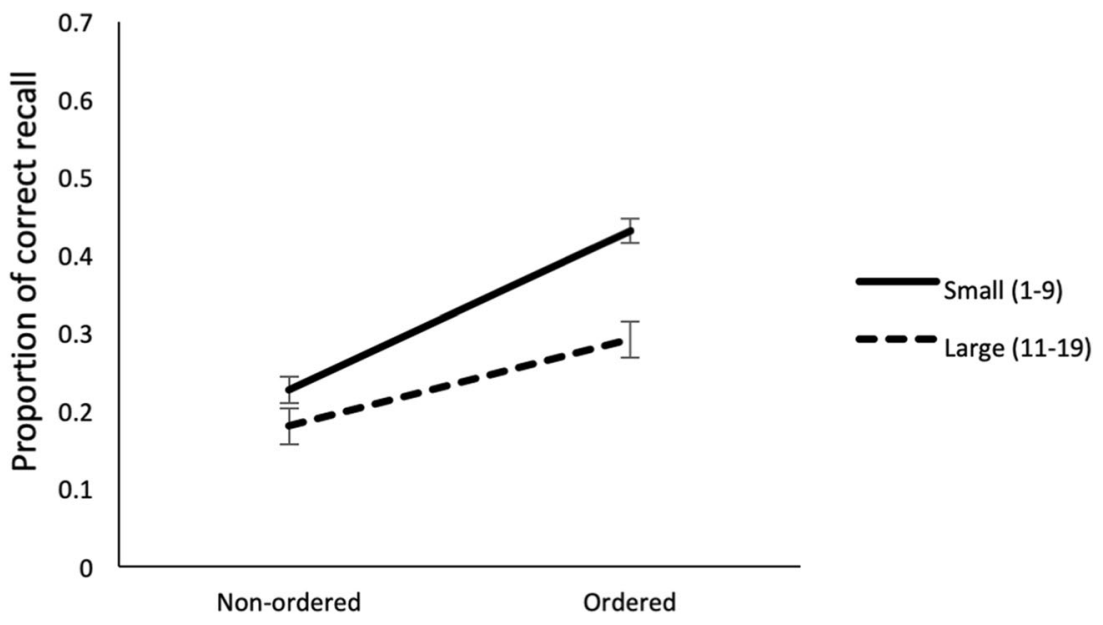

Fig. 2 Interaction between numerical order and range in the immediate serial recall lists. Error bars depict standard errors

counting task. Results further showed that ordered recall uniquely predicted counting skills, even after controlling for non-ordered recall.

The fact that the number sequence order effect steadily increases with age suggests a continuous refinement of the structure of the verbal number sequence in long-term memory while counting is progressively acquired. In line with our findings, Baroody and Price (1983) already observed that some children were able to understand counting principles like the stable order of the number words without being able to apply these principles above a certain range without errors. Similarly, children seem to understand that number words are referring only to specific quantities with order relations between these words before knowing to what quantity each word corresponds (Sarnecka \& Gelman, 2004). Gelman and Meck (1983) showed that children of 3-year-olds could detect order errors in a puppet's counting performance, while they could not yet count in that range, suggesting that young children possess some knowledge about the verbal number sequence before being able to apply this knowledge. The current data extend the findings on children's implicit knowledge about numbers, more specifically concerning the verbal number list without the usually embedded other aspects of counting. The current results provide evidence for the existence of implicit knowledge about the counting list stored in long-term memory before explicit counting is mastered. Independent of the use of numbers in counting and the understanding of ordinality or cardinality, this study demonstrates that the construction of the verbal number sequence in longterm memory starts well before children are able to count explicitly, as implicit knowledge about the number sequence can already be observed in 3-year-olds.

Moreover, the present study provides an easy and sensitive way of assessing this type of implicit verbal number sequence knowledge at different ages. Assessment of this knowledge is important as it represents the progressive development of counting abilities. Existing tools evaluating children's counting abilities typically assess counting skills in general without a specific focus on this very early stage of number knowledge (e.g., TediMath, Van Nieuwenhoven, Grégoire, \& Noël, 2001). These tests thus assess a mixture of knowledge about cardinal relations and number code mastery, which is essential to develop efficient counting skills, but does not allow isolation of selective deficits of verbal number sequence knowledge from other types of numerical deficits. In that framework, the method we developed could be used in the future to assess the implicit knowledge of the counting list in an incidental manner to complement the existing assessment tools and to further study the contribution of that early implicit knowledge to successful numerical learning.

Learning to count implies acquiring arbitrary sequences of number words. Despite preschoolers' limited knowledge of the number list, the current study indicates that preschoolers show superior recall performance for lists of lexical primitives in a conventional order as compared to a scrambled order. This result supports and extends previous data reported by Barrouillet et al. (2010). In their study, preschool children showed increased recall performance for auditory lists of large numbers (e.g., three ninety hundred two) when the numbers form a syntactically coherent structure allowing regrouping of the numbers in a single large number chunk (e.g., three hundred ninety two), although children of this age cannot yet count up to such large numbers. The study by Barrouillet and colleagues indicates that 5 -year-old children already possess implicit syntactic knowledge about the way number labels can be regrouped in order to form a lexically legal large number label. The present study goes beyond that study by showing that younger children also possess implicit knowledge about the ordinal structure and succession of individual small numbers, and this before they have acquired explicit knowledge about the number sequence as revealed by counting-up tasks. These different results can be related more generally to implicit learning abilities that have been documented in children in different domains (Perruchet \& 

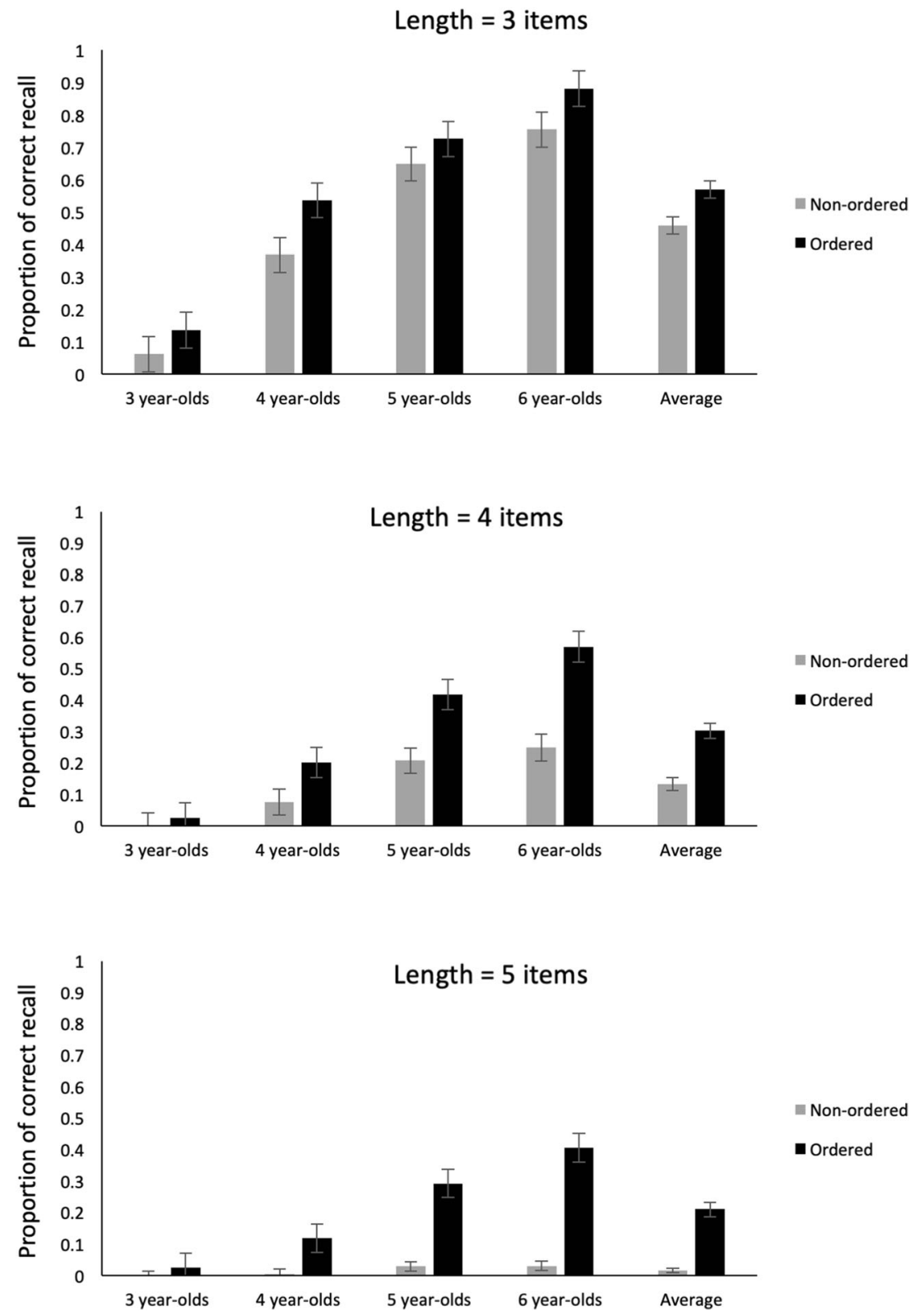

Non-ordered

- Ordered

Fig. 3 Interaction between numerical order, list length, and age group in the immediate serial recall lists. Error bars depict standard errors

Pacton, 2006). For example, Lehtonen and Bryant (2005) as well as Pacton and Fayol (2000) showed that children are sensitive to the frequency of occurrence of letter patterns even before having any knowledge about letters and their meaning. This implicit knowledge is likely to support the explicit acquisition of number and letter knowledge by helping children to distinguish legal from illegal number and letter sequences at a later developmental stage. This is also supported by studies showing that verbal number knowledge is a strong predictor of later arithmetic performance (Koponen, Eklund, \& Salmi, 2018).

In conclusion, the present study used a novel experimental paradigm to specifically target the knowledge of the verbal number sequence in children without involving other aspects of counting. The results provide empirical evidence that, similar to children possessing implicit knowledge about order before cardinal principle is acquired, they also possess an implicit knowledge specifically of the verbal number sequence. We further show that this knowledge is already represented in long-term memory before counting is fully acquired. This incidental assessment paradigm will need to be further examined in future research but we believe it opens a new perspective for the assessment of numerical acquisition in young children.

Acknowledgements We thank the children, their parents, and the schools for their participation. We gratefully acknowledge Mathilde Roch for her help with data collection. This research did not receive any specific grant from funding agencies in the public, commercial, or not-for-profit sectors. 
Data availability Material and data for the current study are available from the corresponding author upon request.

\section{References}

Baroody, A. J., \& Price, J. (1983). The development of the number-word sequence in the counting of three-year-olds. Journal for Research in Mathematics Education, 14(5), 361.

Barrouillet, P., Thevenot, C., \& Fayol, M. (2010). Evidence for knowledge of the syntax of large numbers in preschoolers. Journal of Experimental Child Psychology, 105(3), 264-271.

Benoit, L., Lehalle, H., Molina, M., Tijus, C., \& Jouen, F. (2013). Young children's mapping between arrays, number words, and digits. Cognition, 129(1), 95-101.

Colomé, À., \& Noël, M.-P. (2012). One first? Acquisition of the cardinal and ordinal uses of numbers in preschoolers. Journal of Experimental Child Psychology, 113(2), 233-247.

Cowan, N. (2001). The magical number 4 in short-term memory: A reconsideration of mental storage capacity. Behavioral and Brain Sciences, 24(1), 87-114.

De Smedt, B., Noël, M.-P., Gilmore, C., \& Ansari, D. (2013). How do symbolic and non-symbolic numerical magnitude processing skills relate to individual differences in children's mathematical skills? A review of evidence from brain and behavior. Trends in Neuroscience and Education, 2(2), 48-55.

Fuson, K. C. (1988). Children's counting and concepts of numbers. New York: Springer.

Fuson, K. C., Richards, J., \& Briars, D. J. (1982). The acquisition and elaboration of the number word sequence. In Children's logical and mathematical cognition (p. 33-92). Springer.

Gallistel, C. R., \& Gelman, R. (1992). Preverbal and verbal counting and computation. Cognition, 44(1-2), 43-74.

Gathercole, S. E. (1999). Cognitive approaches to the development of short-term memory. Trends in Cognitive Sciences, 3(11), 410-419.

Gathercole, S. E., Frankish, C. R., Pickering, S. J., \& Peaker, S. (1999). Phonotactic influences on short-term memory. Journal of Experimental Psychology: Learning, Memory, and Cognition, $25(1), 84$.

Gelman, R. \& Gallistel, C. R. (1978). The child's understanding of number. Cambridge, MA: Harvard University Press.

Gelman, R., \& Meck, E. (1983). Preschoolers' counting: Principles before skill. Cognition, 13(3), 343-359.

Gould, P. (2017). Mapping the acquisition of the number word sequence in the first year of school. Mathematics Education Research Journal, 29(1), 93-112.

Hulme, C., Maughan, S., \& Brown, G. D. (1991). Memory for familiar and unfamiliar words: Evidence for a long-term memory contribution to short-term memory span. Journal of Memory and Language, 30(6), 685-701.

Huntley-Fenner, G., \& Cannon, E. (2000). Preschoolers' magnitude comparisons are mediated by a preverbal analog mechanism. Psychological Science, 11(2), 147-152.

Jarlégan, A., Fayol, M., \& Barrouillet, P. (1996). De soixante douze à 72, et inversement: Une étude du transcodage chez les enfants de 7 ans. Revue de Psychologie de l'Education, 1, 109-131.

Izard, V., Pica, P., Spelke, E. S., \& Dehaene, S. (2008). Exact equality and successor function: Two key concepts on the path towards understanding exact numbers. Philosophical Psychology, 21(4), 491-505.

Koponen, T., Eklund, K., \& Salmi, P. (2018). Cognitive predictors of counting skills. Journal of Numerical Cognition, 4(2), 410-428.
Kowialiewski, B., \& Majerus, S. (2018). The non-strategic nature of linguistic long-term memory effects in verbal short-term memory. Journal of Memory and Language, 101, 64-83.

Laski, E. V., \& Siegler, R. S. (2007). Is 27 a big number? Correlational and causal connections among numerical categorization, number line estimation, and numerical magnitude comparison. Child Development, 78(6), 1723-1743.

Le Corre, M., Vandewalle, G., Brannon, E., \& Carey, S. (2006). Revisiting the competence/performance debate in the acquisition of the counting principles. Cognitive Psychology, 52(2), 130-169.

Lehtonen, A., \& Bryant, P. (2005). Doublet challenge: Form comes before function in children's understanding of their orthography. Developmental Science, 8(3), 211-217.

Lyons, I. M., \& Beilock, S. L. (2009). Beyond quantity: Individual differences in working memory and the ordinal understanding of numerical symbols. Cognition, 113(2), 189-204. doi:https://doi.org/10. 1016/j.cognition.2009.08.003

Majerus, S., \& Van der Linden, M. (2003). Long-term memory effects on verbal short-term memory: A replication study. British Journal of Developmental Psychology, 21(2), 303-310.

Majerus, S., Van der Linden, M., Mulder, L., Meulemans, T., \& Peters, F. (2004). Verbal short-term memory reflects the sublexical organization of the phonological language network: Evidence from an incidental phonotactic learning paradigm. Journal of Memory and Language, 51(2), 297-306.

Majerus, S., Perez, T. M., \& Oberauer, K. (2012). Two distinct origins of long-term learning effects in verbal short-term memory. Journal of Memory and Language, 66(1), 38-51.

Majerus, S. (2019). Verbal working memory and the phonological buffer: The question of serial order. Cortex, 112, 122-133.

Martin, R. B., Cirino, P. T., Sharp, C., \& Barnes, M. (2014). Number and counting skills in kindergarten as predictors of grade 1 mathematical skills. Learning and Individual Differences, 34, 12-23.

Mundy, E., \& Gilmore, C. K. (2009). Children's mapping between symbolic and nonsymbolic representations of number. Journal of Experimental Child Psychology, 103(4), 490-502.

Mussolin, C., Nys, J., Leybaert, J., \& Content, A. (2015). How approximate and exact number skills are related to each other across development: A review. Developmental Review Developmental Review, $39,1-15$.

Pacton, S., \& Fayol, M. (2000). The impact of phonological cues on children's judgements of nonwords: The case of double letters. Current psychology letters. Behaviour, brain \& cognition, (2000/1, 1).

Perruchet, P., \& Pacton, S. (2006). Implicit learning and statistical learning: one phenomenon, two approaches. Trends in Cognitive Sciences, 10(5), 233-238.

Pica, P., Lemer, C., Izard, V, \& Dehaene, S. (2004). Exact and Approximate Arithmetic in an Amazonian Indigene Group. Science, 306(5695), 499-503.

Sarnecka, B. W., \& Carey, S. (2008). How counting represents number: What children must learn and when they learn it. Cognition, 108(3), 662-674.

Sarnecka, B. \& Gelman, S. (2004). Six does not just mean a lot: preschoolers see number words as specific. Cognition, 92(3), 329-352.

Van Nieuwenhoven, C., Grégoire, J., \& Noël, M. P. (2001). Test Diagnostique des Compétences de Base en Mathématiques (TediMath). Paris: ECPA.

Wynn, K. (1992). Children's acquisition of the number words and the counting system. Cognitive Psychology, 24(2), 220-251.

Publisher's note Springer Nature remains neutral with regard to jurisdictional claims in published maps and institutional affiliations. 